\title{
On Applications of Ion Chromatography in Analysis of Chemical Substance in Food and Drink and Food Safety Problems
}

\author{
Xinlu Lian \\ School of Chemistry, Sun Yat-sen University, Guangzhou, China
}

Keywords: Ion chromatography, Food safety, Research progress, Food, Drink

\begin{abstract}
The harmful substance in food and drink often brings the harm of different degrees to people's health. The lack of effective substance in health food cannot meet the needs of people's supplementary nutrients. Therefore, it is very important to detect the amount of harmful substance in common food and drink and the amount of effective substance in health food. Through the means of example and contrast, the paper expounds the health threats brought about super-standard harmful substance in food and drink and detects the varied food and drink samples by the analysis technology of ion chromatography. When ion chromatography is applied in the detection of relative substance in food and drink, the detection limits are always low and the sensitivities are always high. It also has the features of high effectiveness and low cost. In addition, the current development bottleneck of ionic chromatography is also discussed, and the future development prospect is also predicted.
\end{abstract}

\section{Introduction}

Food and drinking water are the basis for human beings and all other creatures on the planet to survive. How to get clean drinking water and eat healthy food is the most important concern of all people. However, the problems of food and drinking water pollution and their direct diseases are common in various media reports. Among them, the WHO highlighted the importance of food safety at the Second International Nutrition Conference in Rome in November 2014 and appealed to all governments around the world to focus food safety as a public health focus - the formulation of policy and regulatory frameworks and the establishment and implementation of effective food safety departments. It plays an important role in ensuring that food producers and suppliers in the food chain operate responsibly and provide consumers with safe food. As a result, people are eager to know whether the rice, pasta, even candy, biscuits and other leisure foods are safe and reliable. Then we need to know what substances in the food and drinking water threaten the health of the human body and know how to use scientific methods to detect and analyse them.

To detect the content of related chemicals in food and drinking water sensitively, accurately and efficiently, the commonly used method is ion chromatography (IC). It is a good method to detect the content of inorganic ions and food additives in drinking water because of its strong selectivity, high sensitivity and ability to detect many kinds of yin and yang ions at the same time.

\section{Overview of ion chromatography}

With the increasing demand for food and drinking water analysis, many modern analytical methods have been developed over the past few decades, including the atomic absorption spectroscopy (AAS), inductively coupled plasma-atomic emission spectroscopy (ICP-AES), and inductively coupled plasma-mass spectrometry (ICP-MS) and so on. Besides, some electrochemical analysis methods are also included. However, these methods are often limited or sensitive, or have spectral and chemical interference, which are limited in the field of food and drinking water detection. Ion chromatography is a good solution to these problems.

Ion chromatography is a technology that can detect organic acids, bases and inorganic anion and cation in substances. Since 1970s, this technology has developed rapidly through the analysis of complex samples of ionic compounds. As early as 1971, the Hamish Small team first reported ion 
chromatography. In 1975, he reported in Analytical Chemistry magazine that IC technology can continuously monitor eluent through ion exchange separation conductance. In 1984, U.S. EPA and ASTM reported the method of water analysis and detection using IC technology. In 1998, automatic sampling technology was born. Now, IC technology has been widely used, which can be used in the qualitative and quantitative analysis of carboxyl (sulfur) ester (anhydride), rare earth elements, heavy metals, transition metals and common inorganic Yin and yang ions, and has not stopped the pace of development.

Ion chromatography is based on the principle of ion exchange and belongs to the scope of the liquid chromatography separation mode. The ion chromatography system mainly includes several parts of the "leaching liquid reservoir, pump, sampling valve, separator, detection system, control and data processing system". The ion chromatography can be divided into high efficiency ion chromatography (HPIC) and high efficiency ion exclusion chromatography, according to the different mechanism of different chemical substances in the separation samples. Analytical method (HPICE) and mobile phase ion chromatography monitoring analysis (MPIC). Different methods have different applications, as shown in Table 1.

Table 1. Ion chromatography and its application fields

\begin{tabular}{c|c}
\hline Name of Ion chromatography & Application fields \\
\hline HPIC & Hydrophilic zwitterion and carbohydrate \\
\hline HPICE & Inorganic weak acid, organic acid and amino acids \\
\hline MPIC & Hydrophobic zwitterion \\
\hline
\end{tabular}

In the process of food testing, ion chromatography has good selectivity, and it can distinguish the composition of many chemical properties in food, which is mainly reflected in the increase of monitoring range in the washing liquid of strong acid (alkali). After power on, IC can also detect low concentration ions accurately. In addition, another significant advantage of IC is that it has high detection efficiency, and can simultaneously detect anions, cations and cyanide ions in the solution, or anions, cations and sugars, and have strong tolerance to the $\mathrm{pH}$ of the solution.

In summary, it is sensitive, fast and accurate to detect harmful chemicals in food and drinking water by ion chromatography.

\section{Sample preparation method}

Different foods, such as meat products, pills, pills and milk powder, and so on, have different characteristics. If you want to use IC to analyse, you must go through the sample pre-treatment process. Naturally, different kinds of foods have different sample pre-treatment methods. Finding and using the appropriate sample pre-treatment methods will make the following analysis more than half the effort, and enhance and improve the accuracy, sensitivity and efficiency of the detection. The sample characteristics that need to be pre-treated without direct injection include: 1) the physical state and shape of the sample cannot be injected directly (i.e., non-solution state); 2) there are obvious components of interference detection results in the sample; 3) the content of the sample is too low to exceed the accuracy of the existing testing methods and instruments. (The original document is: the analyte concentration is out of the detection ability of the corresponding apparatus or methods.) The common pre-treatment methods include dissolution, homogenization, extraction, evaporation, separation, chemical derivation and standardization. In practical application, we should select suitable pre-treatment methods for sample preparation according to the different characteristics of the food. 


\section{Application examples of ion chromatography in food detection}

\subsection{Pickled food and instant food}

Pickled food and instant food are widely used. Common products include instant noodles, luncheon meat, Korean pickles, canned fish and so on, which can be easily purchased in various supermarkets. This kind of food will be greatly welcomed when time is urgent and cannot spend too much time to prepare meals, such as rescue and relief or war, or in urban life for the sake of convenience and convenience. But people often neglect the potential danger of this kind of food.

As the pickled fast food is solid, ultrasonic extraction, solid phase dispersion and solid phase extraction are used to extract samples. For example, in the determination of nitrate and nitrite in kimchi, a high-speed centrifuge solid phase extraction column is used to carry out the sample. Sometimes, researchers need to exclude the interference of other ions, such as the detection of nitrite content in salted fish to avoid the interference of $\mathrm{Cl}^{-}$, the IC-Ag preconditioning column is used to eliminate.

When the leaching conditions are selected, the concentration of the eluent (commonly used $\mathrm{NaHCO}_{3}$ and $\mathrm{Na}_{2} \mathrm{CO}_{3}$ solution or two solutes in a certain proportion) will affect the balance of ion exchange and the retention of ions. The higher the concentration of the leached ion, the stronger the ability of the leaching solution to replace the ions from the stationary phase of the chromatographic column and the shorter the elution time of the solute ions. The short retention time is not conducive to the separation of interfering ions from $\mathrm{NO}_{2}{ }^{-}$. Therefore, it is necessary to find a concentration of eluent which can make $\mathrm{NO}_{2}{ }^{-}$and other ions have the best separation effect.

Chen Junxi found that the relative standard deviation (RSD) of five consecutive measurements of $\mathrm{NO}_{2}{ }^{-}$and $\mathrm{NO}_{3}{ }^{-}$was less than $3 \%$, and the recovery rate was greater than $80 \%$ when the samples were low $\mathrm{Cl}^{-}$of kimchi. When using salted fish purchased in Guangzhou aquatic product market, the detection limit is $0.2 \mathrm{mg} \cdot \mathrm{kg}^{-1}$, and the recovery rate is not less than $83 \%$. This indicates that the method is accurate, stable and sensitive for the detection of nitrite and nitrate in salted food. The test results are representative.

\subsection{Analysis of effective substance in health food}

Calcium is an important nutrient indispensable to the human body. It plays an important role in the growth and development of human body and participates in the whole life process. Proteins that bind to $\mathrm{Ca}^{2+}$ are useful in muscle, nerve, body fluid and bone. It is the main inorganic component of human bone and tooth. It is also the essential element for nerve transmission, muscle contraction, blood condensation, hormone release and milk secretion, which accounts for about $1.4 \%$ of the body's mass. The benefits of calcium supplement and the harm of calcium deficiency are found in the media reports. Many people have the habit of supplementing calcium. All kinds of high calcium food and health care products are becoming more and more popular and have a broad and attractive market. At the same time, some illegal businesses use people's blind calcium supplements, lack of professional knowledge and other shortcomings, the calcium content of the products sold did not meet the advertising requirements. Therefore, finding an analytical method conducive to the analysis of calcium tablets and calcium containing beverages, such as high calcium milk, will be conducive to market regulation and public choice of products.

Calcium standard solution is simple and easy to get and can be accurately made into standard series in ordinary laboratories, and the linear condition is ideal. The pre-treatment method of calcium sample is not very difficult. After the mixed acid of $\mathrm{HNO}_{3}$ and $\mathrm{HClO}_{4}$ is digested with pure water, the sample can be directly injected after $0.45 \mathrm{U}$ M filter membrane. The minimum detection limit of calcium ion is only $0.02 \mathrm{mg} \mathrm{L}^{-1}$, and the recovery rate of calcium ion is between 95.0\%-103.0\% and the quality control requirement of China is 11 times. In addition, when adding $\mathrm{Cu}^{2+}, \mathrm{Mg}^{2+}, \mathrm{K}^{+}, \mathrm{NH}^{4+}, \mathrm{Al}^{3+}, \mathrm{Fe}^{3+}$ plasma to the sample, the relative standard deviation of the six experimental results is $0.5 \%-2.0 \%$.

Thus, it can be seen that the use of DX-120 ion chromatograph to determine calcium content in food by ion chromatography is simple, reliable and not easily interfered by other ions. Moreover, 
the method is simple and efficient, and can be widely applied to the determination of calcium content in foods.

\section{Application examples of ion chromatography in drink detection}

\subsection{Detection of chemicals in drinking water}

We know that the tap water used in normal city needs a series of complicated process and transportation. The drinking water that we rely on will go into the thousands of households along the indoor faucets. In rural areas, people also use the way of well drilling to get natural surface water. If the water is contaminated, or the transport process is polluted, then our drinking water will be difficult to reach the health standards of the authorities, endangering the health of the people, and in serious cases the population may be affected.

The chemical composition of everyday polluted drinking water includes all kinds of inorganic ions. Cations such as $\mathrm{Na}^{+}, \mathrm{Li}^{+}, \mathrm{K}^{+}, \mathrm{Mg}^{2+}, \mathrm{Ca}^{2+}$ and other metal ions, the excessive intake of metal ions can lead to different results, including kidney stones, liver damage, and so on. Anions such as $\mathrm{F}^{-}, \mathrm{Cl}^{-}, \mathrm{NO}^{3-}, \mathrm{Br}^{-}, \mathrm{PO}_{4}{ }^{3-}, \mathrm{SO}_{4}{ }^{2-}$ and so on, their high content will be the body metabolism disorder, skin burns, respiratory inflammation and other symptoms, and a strong carcinogen. In addition, in the process of production of tap water, the disinfectant and its by-products (including chlorine, bleaching powder and ozone) may also be inflow into the water pipe. Their detection methods are also worthy of attention.

There is almost no need for complex sample pre-treatment before drinking water is tested. Generally, the water sample is removed for a period after the ultrasonic bubbles are removed. The test requirements can be achieved by vacuum filtration through the filter membrane before sampling.

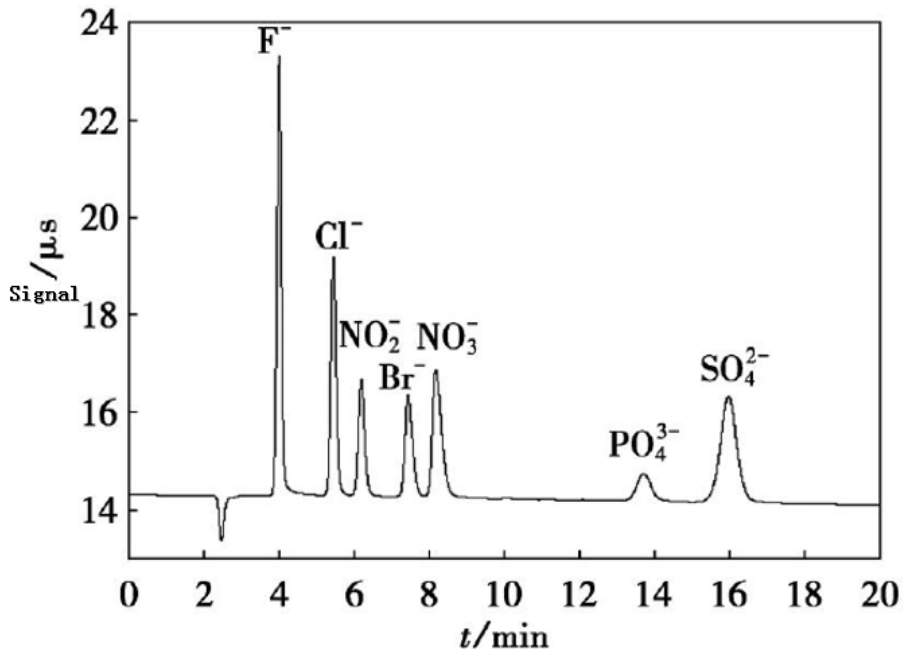

Figure 1. Chromatograms and peak positions of all anions in mixed solution

Wu Ying and others from Shenyang Normal University used the mixed anion standard solution to enter the sample at the specific concentration $\mathrm{Na}_{2} \mathrm{CO}_{3}$ and $\mathrm{NaHCO}_{3}$ as drenching solution in the anion detection section. The ion chromatography 1 shows that the anion separation effect is good and can be detected at the same time. They were quantified by the anionic solution prepared by a certain gradient standard. After the linear equation was obtained, the samples were then injected into the IC chromatograph to analyse the content. When the cations were detected, the IC technology was used by Zheng Shaona of Guangdong environmental monitoring centre to determine the alkali metal, alkaline earth metal and ammonium ions in drinking water at the same time.

Chlorine disinfection is the main way to disinfect urban tap water in China, and some of them are sterilized by $\mathrm{ClO}_{2}$ and ozone. Disinfection by-products produced in the disinfection process of drinking water are harmful, including halo acetic acid and three halo methane, which can inhibit cell apoptosis (proliferation), influence sugar metabolism and induce tumor disease and so on. 
Among them, haloacetic acid has higher content of two chloroacetic acid (DCAA) and three chloroacetic acid (TCAA), and its unit concentration has greater risk of cancer. At present, the determination of DCAA and TCAA in China is mainly by gas chromatography. This method has a tedious pre-treatment and extraction derivation process, and its application is limited. Liu Yongjian and other scholars applied IC method to simultaneously detect nine halogenated acetic acids in drinking water. The detection limit reached $\mu \mathrm{g} / \mathrm{L}$ level, which was also accurate and convenient.

\subsection{Detection of food additives in beverages}

People can easily buy various kinds of flavoured beverages on the market. Careful observation of the ingredients list of beverage bottles reveals that in addition to the use of water, sugar and syrup, various food additives are also used in beverages. The effects of these additives include: enhancing the sensory quality of the beverage, improving the stability of the beverage, improving the nutritional value of the beverage, preventing the corruption and deterioration of the beverage, increasing the variety and convenience of the beverage and so on. A certain amount of food additives, such as citric acid, aspartame, cyclamate, sativoside, potassium sorbate and so on, not only have no toxicity and harm, but also make the beverage unique, more durable, and arouse the consumer's desire to buy. But excessive food additives are mainly reflected in the disturbance of the body's metabolism, increase the risk of heart disease and diabetes, and there is a potential carcinogenic risk.

Because the sensitivity of the conductivity detector to organic acids is relatively high, the detection of trace organic acids in food is preferably by ion chromatography. The contents of sodium cyclamate, sorbic acid, benzoic acid, benzoic acid, anasai and saccharin sodium in the beverage were measured by ion exchange and conductivity detection by Wang Aiyue, the Henan Centre for Disease Control and prevention. They use Ion Pac AS19 4 × $250 \mathrm{~mm}$ as analysis column, Ion Pac AS19 4 x $50 \mathrm{~mm}$ as protection column, $18 \mathrm{mM}, \mathrm{KOH}$ as drenching liquid, $1.0 \mathrm{ml} / \mathrm{min}$ flow rate, obtained the linear excellent standard curve, the final detection limit reaches the $\mu \mathrm{g} / \mathrm{L}$ level. The standard deviation of repeated measurement is small, and the recovery rate is high. In addition, they found that the sweeteners such as citric acid, tartaric acid and aspartame would not interfere with the chromatographic conditions. This indicates that this method can be widely applied to the determination of additives in mainstream beverages without eliminating interference from related organic matters.

\subsection{Detection of nitrite and nitrate in milk and other dairy products}

Milk is a common daily drink. Some businesses will add nitrite or nitrate as a colour developing agent and preservative in the process of separating and preserving milk. In addition, the storage time of raw milk is too long, the feed of the cows, the nitrite in the water, and so on, will also make the finished milk (sub) nitrate in the hands of the consumers exceed the standard, endangering the health of the human body.

The methods for the determination of nitrate in dairy products include gas chromatography, $\mathrm{AgNO}_{3}$ selective electrode for direct potential of nitrate in milk powder, automatic cyclic septum determination, cadmium column reduction and spectrophotometric analysis, etc. their common characteristics are the shortcomings of time-consuming operation and non-adaptation to large batch samples, and the use of Cd powder. It doesn't conform to the idea of green chemistry. In this way, ionic chromatography has its own advantages and will have wide application space in the future.

One of the more classical methods of ion chromatography is to use $\mathrm{Na}_{2} \mathrm{CO}_{3}$ and $\mathrm{NaHCO}_{3}$ as the drenching solution. After adding $\mathrm{CH}_{3} \mathrm{COOH}$ and $\mathrm{NaOH}$ to remove the insoluble organic compounds, such as protein and lipid, the samples are directly injected into the sample. Zhou Hong reported that the detection limit of this method was as low as $0.15 \mathrm{mg} / \mathrm{kg}$, and its sensitivity was 100 times of that of the national standard method $(1.5 \mathrm{mg} / \mathrm{kg})$. The recovery rate of fresh milk and infant milk powder was between $89.5 \%-112.0 \%$.

Later, Cui Xiaoqing and others found that $\mathrm{CH}_{3} \mathrm{COOH}$ precipitates were adsorbed on the chromatographic column at the time of detection, resulting in irreversible damage to the chromatographic column. To improve the method, acetonitrile was used in the pre-treatment of milk 
and water samples, and the supernatant was centrifuged to remove the precipitation, and then 0.22 $\mu \mathrm{m}$ organic phase filter membrane was used to filter the back sample. Acetonitrile can produce fine protein precipitation, high precipitation efficiency, and can obtain a cleaner sample matrix to reduce the contamination and damage of impurities in the IC system.

On the whole, IC technology has high recovery rate and the detection limit is at the level of $\mu \mathrm{m} / \mathrm{L}$, which is a good method.

\section{Summary and prospect}

Ion chromatography has been a frontier and popular analytical technology after a long period of development. Through the analysis of the above examples, the technology is quick to analyse the common anions such as $\mathrm{NO}^{2-}, \mathrm{NO}^{3-}, \mathrm{Cl}^{-}$and other cations, such as $\mathrm{Ca}^{2+}$ and $\mathrm{Na}^{+}$, and can be separated by high efficient separation column. The advantages of this method are high sensitivity (the detection limit is in the $\mu \mathrm{g} / \mathrm{L}$ level), and the selectivity is good (using appropriate separation methods, separation columns and monitoring methods can be realized), and a variety of ionic compounds can be analysed at the same time.

However, in practical applications, ion chromatography still has room for improvement. For example, ion chromatography is inseparable from the pre-treatment steps of samples. For solid food, such as food, solid dispersion, solid phase extraction and ultrasound can be used to form the solution and then sample. The detection of liquid substances and general drinking water can be directly injected into sample or after concentration and then re-entered (when the sensitivity is not high or only trace amount of material to be measured), and milk and other fluid containing lipid and protein need to be precipitated and centrifuged. The service life of chromatographic column is limited, and the cost is high. Sometimes, to get good test results, it is a great expense to constantly replace high-quality fillers. In addition, if a mobile phase, such as strong acid and strong alkali, has been reacted with the remnants of the sample after the chromatography column, the precipitate will block the exit of the column, cause pollution and influence the reuse of the column. To obtain stable and accurate results, the ion chromatography column needs to be balanced for a long time. There are many problems in the experiment, such as the large displacement of the cation peak and the instability of the peak type, which often affect the experimental results and accuracy of the detection.

The above points are common development bottlenecks of IC technology in recent years, which are mainly reflected in the development of chromatographic columns and fillers. If the ion chromatography technology is to be applied more widely, we should consider getting rid of the above constraints. We should reduce the production cost and improve the efficiency and accuracy of column utilization. In this way, the IC technology will have a broader development platform.

\section{References}

[1] Weber W, Wagner R, Streipert B, et al. Ion and gas chromatography mass spectrometry investigations of organophosphates in lithium ion battery electrolytes by electrochemical aging at elevated cathode potentials[J]. Journal of Power Sources, 2016, 306: 193-199.

[2] Kraft V, Weber W, Grützke M, et al. Study of decomposition products by gas chromatographymass spectrometry and ion chromatography-electrospray ionization-mass spectrometry in thermally decomposed lithium hexafluorophosphate-based lithium ion battery electrolytes[J]. Rsc Advances, 2015, 5(98): 80150-80157.

[3] Michalke B, Witte H. Characterization of a rapid and reliable method for iodide biomonitoring in serum and urine based on ion chromatography-ICP-mass spectrometry[J]. Journal of Trace Elements in Medicine and Biology, 2015, 29: 63-68.

[4] Shchukina O I, Zatirakha A V, Smolenkov A D, et al. Anion exchangers with branched functional ion exchange layers of different hydrophilicity for ion chromatography[J]. Journal of Chromatography A, 2015, 1408: 78-86. 
[5] Barron L, Gilchrist E. Ion chromatography-mass spectrometry: a review of recent technologies and applications in forensic and environmental explosives analysis[J]. Analytica chimica acta, 2014, 806: 27-54.

[6] Park S H, Haddad P R, Talebi M, et al. Retention prediction of low molecular weight anions in ion chromatography based on quantitative structure-retention relationships applied to the linear solvent strength model[J]. Journal of Chromatography A, 2017, 1486: 68-75.

[7] Ni C, Zhu B, Wang N, et al. Simple column-switching ion chromatography method for determining eight monosaccharides and oligosaccharides in honeydew and nectar[J]. Food chemistry, 2016, 194: 555-560.

[8] Rajski Ł, Díaz Galiano F J, Cutillas V, et al. Coupling Ion Chromatography to Q-Orbitrap for the Fast and Robust Analysis of Anionic Pesticides in Fruits and Vegetables[J]. Journal of AOAC International, 2018, 101(2): 352-359.

[9] Pyschik M, Schultz C, Passerini S, et al. Aging of cations of ionic liquids monitored by ion chromatography hyphenated to an electrospray ionization mass spectrometer[J]. Electrochimica Acta, 2015, 176: 1143-1152.

[10] Wang J, Christison T T, Misuno K, et al. Metabolomic profiling of anionic metabolites in head and neck cancer cells by capillary ion chromatography with Orbitrap mass spectrometry[J]. Analytical chemistry, 2014, 86(10): 5116-5124.

[11] Gilchrist E S, Nesterenko P N, Smith N W, et al. Organic solvent and temperature-enhanced ion chromatography-high resolution mass spectrometry for the determination of low molecular weight organic and inorganic anions[J]. Analytica chimica acta, 2015, 865: 83-91.

[12] Sánchez M I G, McCullagh J, Guy R H, et al. Reverse Iontophoretic Extraction of Metabolites from Living Plants and their Identification by Ion - chromatography Coupled to High Resolution Mass Spectrometry[J]. Phytochemical Analysis, 2017, 28(3): 195-201.

[13] Song M, Wang J, Chen B, et al. A facile, nonreactive hydrogen peroxide (H2O2) detection method enabled by ion chromatography with UV detector[J]. Analytical chemistry, 2017, 89(21): 11537-11544.

[14] Karasinski J, Bulska E, Wojciechowski M, et al. High precision direct analysis of magnesium isotope ratio by ion chromatography/multicollector-ICPMS using wet and dry plasma conditions[J]. Talanta, 2017, 165: 64-68.

[15] Miyake Y, Tokumura M, Iwazaki Y, et al. Determination of hexavalent chromium concentration in industrial waste incinerator stack gas by using a modified ion chromatography with post-column derivatization method[J]. Journal of Chromatography A, 2017, 1502: 24-29. 